\title{
License Plate Character Recognition System using Neural Network
}

\author{
Anuja P. Nagare \\ Thadomal Shahani Engineering College. \\ University of Mumbai, \\ Mumbai-400 032, India.
}

\begin{abstract}
Intelligent Transportation System (ITS) has become an integral part of the Transportation Industry these days and it consists of License Plate Recognition (LPR) System. License Plate Recognition is also called Car Plate Recognition (CPR) or Automatic Number Plate Recognition (ANPR) System. In LPR System, when a vehicle steps over magnetic loop detector it senses car and takes image of the car, following image preprocessing operations for improvement in the quality of car image. From this enhanced image, license plate region is recognized and extracted. Then character fragmentation/segmentation is performed on extracted License Plate and these segmented characters are recognized using Neural Network in this paper.
\end{abstract}

\section{General Terms}

Image Processing, Neural Network.

\section{Keywords}

LPR, Character Segmentation, Character Recognition.

\section{INTRODUCTION}

Transportation is a rapidly evolving industry. Recently, there has been much tremendous advancement in this industry most notably Intelligent Transportation System (ITS). License Plate Recognition (LPR) is an integral part of ITS. The popularity of License Plate Recognition System is mainly because of its successful applications in Traffic congestion and monitoring, Revenue control related to road usage, Campus security Systems, Access Control Systems, etc [1][2].

License Plate Recognition consists of three main phases:

\section{License Plate Detection/Extraction,}

\section{Character Segmentation and}

3. Character Recognition.

In the paper, still pictures of vehicles are used as input to the System. In the first step image enhancement is performed using Contrast stretching followed by Tophat-Bothat Transform. Then in the next step Sobel Operator is used for edge detection. After edge detection series of morphological operations are performed in order to detect the license plate. Then character segmentation is done using line scanning technique, scanning is done from left to right of the plate. After Character Segmentation, feature extraction is performed to obtain the unique features of every character.
This paper uses two Neural Network techniques for character recognition, one is Back Propagation Neural Network and other one is Learning Vector Quantization Neural Network. Their results are compared based upon their perfection in the character recognition. It is observed that the character recognition results obtained using Learning Vector Quantization Neural Network (LVQ NN) is better than the character recognition results obtained by using Back Propagation Neural Network (BP NN). The efficiency of the System can be further improved by increasing the number of fonts for training Neural Networks.

Figure 1 shows the model of License Plate Recognition System used in this paper.

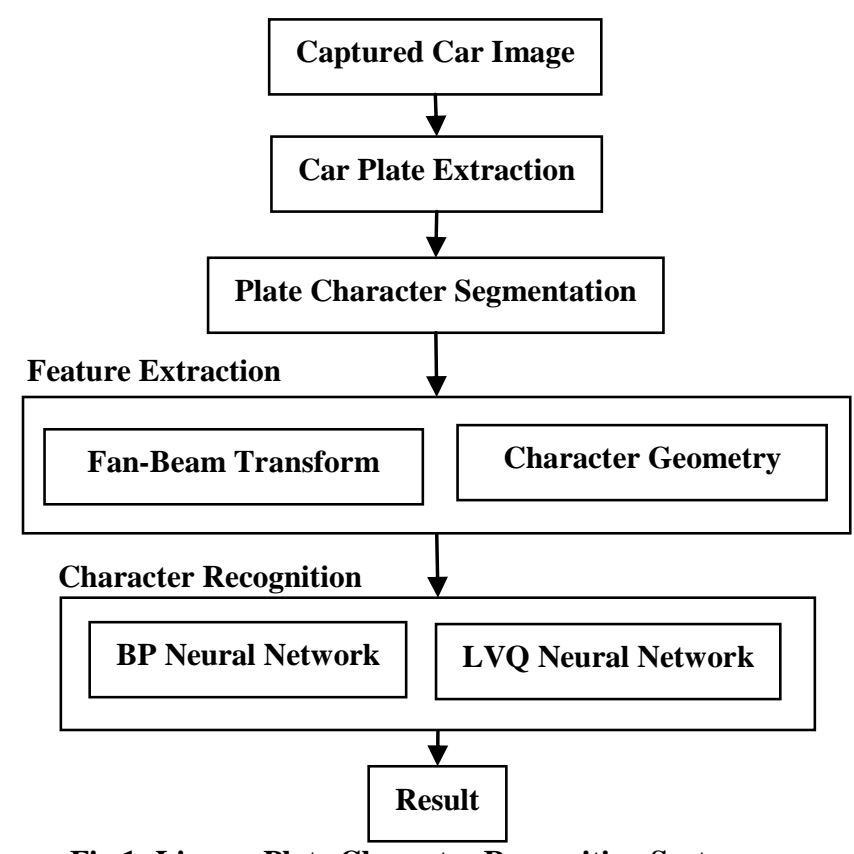

Fig 1: License Plate Character Recognition System

The remainder of this paper is organized as follows: Section 2 introduces Plate Extraction technique. Section 3 describes Character Segmentation, Section 4 explains Feature Extraction of segmented character images. Section 5 presents Character Recognition module of LPR system. Finally, conclusion is drawn of the work and possible future work is discussed in section 6 . 


\section{PLATE EXTRACTION}

To enhance quality of car image for better results in further operations on the Number plate, Image Preprocessing operations are performed on the original car image. "A License Plate Locating Method Based on Tophat-Bothat Changing and Line Scanning" [3], introduces the whole process of image preprocessing and of plate region detection. The captured images are converted to digital form using an image collection card. This image is then converted to a gray scale image the next step is image pre-processing for which Top-hat and Bot-hat technique is used. This technique improves the overall contrast of the image. After enhancing image, License Plate Detection is done using various morphological operations [4].

Figure 2 shows the flowchart for License Plate extraction operations.

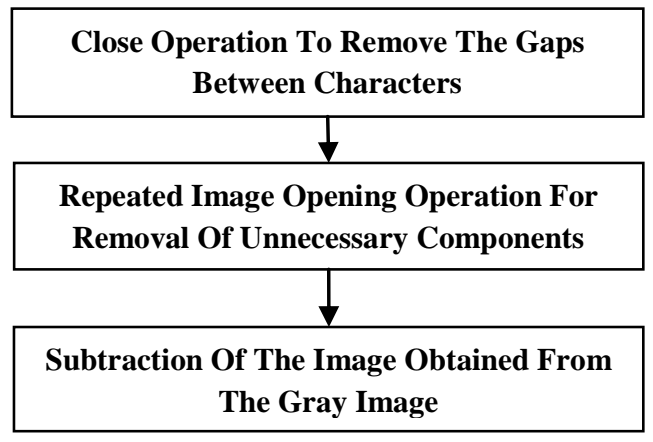

Fig 2: Plate Extraction Operations

Figure 3 shows, Plate Extraction in three steps. First original car image is taken as input; image preprocessing is done on this image and in the last step plate is extracted from preprocessed image.

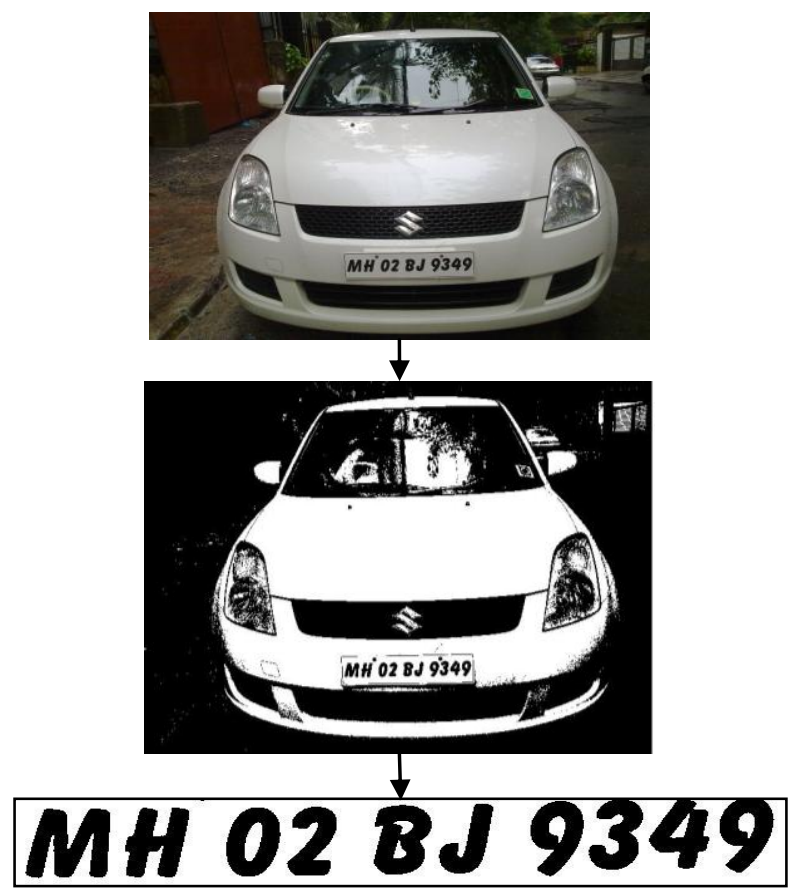

Fig 3: Plate Extraction

\section{CHARACTER SEGMENTATION}

The License plate obtained from Plate Extraction has characters is gray-scale. To obtain segmented characters, first plate image is converted into binary image. Then 'Lines' Function is used to divide text on the number plate into lines, which uses 'clip' function. 'Clip' function crops black letter with white background. After cropping image, resizing is done and same operation is repeated on the cropped image. This process is followed till all the characters are segmented [5].

Figure 4 shows, License Plate Character Segmentation results.

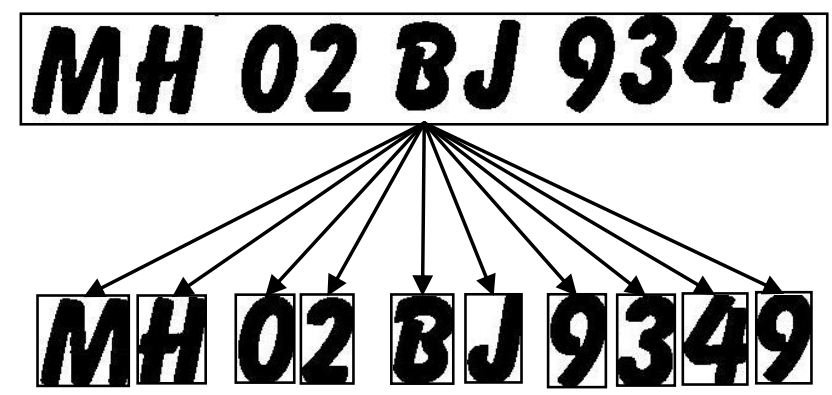

Fig 4: Character Segmentation

\section{FEATURE EXTRACTION}

In this paper, as Neural Network is used for character recognition, Feature Extraction is an important step for training and simulating the Neural Network. Feature Extraction is performed on each segmented character. Two feature extraction techniques are tried for training and simulating Neural Network. One is Fan-beam Transform and other technique is based on Character Geometry.

Fan-beam Transform is used for computing an alternative mathematical representation of an image using Fan-beam projections [6]. Fan-beam function computes projections of an image matrix along specified directions. Projection of a two dimensional function $f(x, y)$ is a set of line integrals. The Fan beam function computes the line integrals along paths that radiate from a single source, forming a fan shape. To represent an image, the Fan-beam function takes multiple projections of the image from different angles by rotating the source (angle is assumed to be $10^{\circ}$ ) around the centre of the image. The distance between the fan-beam vertex and the center of rotation (center pixel of the image) is fixed for all projections (distance is assumed to be 200 pixels), this distance must be large enough to ensure that the fan-beam vertex is outside of the image at all rotation angles. There are 15 sensors spaced equally along a circular arc for each projection. To cover 360 degrees with $10^{\circ}$ rotation angle 36 projections are required. Thus column of fan beam data (features) of the image is 540 sensor samples.

"A Feature Extraction Technique Based on Character Geometry for Character Recognition" [7] describes a geometry based technique for feature extraction. These features are based on the basic line types that form the character skeleton. The column of features for Character Geometry based feature extraction has 55 values.

It is a general observation in any neural network system that, if the network is trained with more number of features the simulation accuracy increases. Numbers of features are more in 
fan-beam feature extraction, Character Recognition accuracy is better for fan-beam feature extraction than Character Geometry technique, as there are 55 features in Character Geometry feature extraction and 540 features in Fan-beam feature extraction.

\section{CHARACTER RECOGNITION}

This step is the main part of the system and is called as Character Recognition step, where segmented characters are recognized. Character Recognition is also called as Optical Character Recognition (OCR)[8][9]. Traditional methods used for number plate recognition were OCR "Optical Character Recognition" and "Formula Based Recognition". As Neural Network" is an intelligence engine, it ensures greater accuracy rate along with better recognition speed [10]. Two learning based neural network techniques are used for character recognition: BP ANN (Back Propagation Artificial Neural Network) and LVQ NN (Learning Vector Quantization Neural Network), after finding out characters of the plate by these two methods, voting can be performed to find the best method based upon the time taken and accuracy in the output of the BPNN and LVQNN [11][12][13][14].

\subsection{Back Propagation Neural Network}

BP (Back Propagation) Neural Network is a supervised neural network, with three layers input layer, output layer and hidden layer [15]. Learning rate used for training the BPNN is 0.09 and transfer function is tansig.

The design of BP NN has 540 input nodes for features of each character, 36 hidden nodes and 36 output nodes.

\subsection{Learning Vector Quantization Neural Network}

Learning Vector Quantization (LVQ) is a hybrid network. In this paper LVQ uses supervised learning to form classification [16][17][18]. In LVQ network each neuron in the first layer is assigned to a class, each class is assigned to one neuron in the second layer. There are three basic steps in LVQ algorithm:

1. Initialization,

2. Competition and

3. Learning.

LVQ NN combines competitive learning with supervision. Target vector is in log sigmoid form (identity matrix). Learning rate is 0.01 for training the network.

Table 2 shows the results of character recognition using Back Propagation Neural Network (BPNN) and Learning Vector Quantization Neural Network(LVQ NN).

Table 2. Results of Character Recognition

\begin{tabular}{|c|c|c|c|}
\hline NN & $\begin{array}{c}\text { Numerical } \\
\text { Rate }\end{array}$ & $\begin{array}{c}\text { Alphabetic } \\
\text { Rate }\end{array}$ & Accuracy \\
\hline BP NN & $70 \%$ & $62 \%$ & $66.67 \%$ \\
\hline LVQ NN & $90 \%$ & $96.15 \%$ & $94.44 \%$ \\
\hline
\end{tabular}

\section{CONCLUSION}

In this paper, method for character recognition of license plate image based on two Neural Network techniques and two feature extraction approaches are proposed. It is observed that, as fan beam feature extraction method has more features for training the neural network thus its simulation accuracy is higher. Using Fan-beam for feature extraction Back Propagation and Learning Vector Quantization Neural Networks are trained. From Table 2 it can be concluded that Recognition accuracy of LVQNN is higher than BPNN.

Thus the further research can be focused on exploring other feature extraction methods which can be used for training the neural network. Also more number of fonts can be used for training the network for improving the accuracy of the character recognition.

\section{ACKNOWLEDGMENTS}

I wish to express my sincere thanks and deep sense of gratitude to respected mentor and guide Ms. Shalini Bhatia, Assistant Professor in Computer Engineering Department of Thadomal Shahani Engineering College., for the technical advice, encouragement and constructive criticism, which motivated to strive harder for excellence.

\section{REFERENCES}

[1] Lee J. Nelson. Electro-Optical Technologies [Online]. Available: "http://www.photocop.com/recognition.htm."

[2] Hsien-Chu WU, Chwei-Shyong TSAI, and Ching-Hao LAI, "A License Plate Recognition System in eGovernment", In proc. of An International Journal, 2004, Vol.15, No.2, pp.199-210.

[3] P G Hou, J Zhao and M Liu, "A License Plate Locating Method Based on Tophat-bothat Changing and Line Scanning", In Proc. of International Symposium on Instrumentation Science and Technology, 2006, pp. 431436.

[4] Antonio Albiol, Jose Manuel Mossi, Alberto Albiol, Valery Naranjo, "Automatic License Plate Reading Using Mathematical Morphology", In proc. of Spanish Ministry of Science and Technology, 2002.

[5] Danilo Octavio[Online]. Available: "http://wwweurope.mathworks.com/matlabcentral/fileexchange/18169optical-character-recognition-ocr", 2009.

[6] Kak, A.C., \& Slaney, M., "Principles of Computerized Tomographic Imaging”, IEEE Press, NY, 1988, pp. 92-93.

[7] Dinesh Dileep, "A Feature Extraction Technique Based On Character Geometry For Character Recognition”, In proc. of Department of Electronics and Communication Engineering, Amrita School of Engineering, pp. 1-4.

[8] Jimmy Wales, Larry Sanger[Online]. Available : "http://en.wikipedia.org/wiki/Optical_character_recognitio n”, 2001.Z

[9] Michael Hogan,John W. Shipman, “OCR (Optical Character Recognition): Converting paper documents to text" In proc. of New Mexico Tech Computer Center, 2008, pp.1-4. 
[10] cctvx - cetv [Online]. Available: "http://www.dbeechassociates.com/anpr.pdf."

[11] Jianlan Feng Yuping Li Mianzhou, "The Research of Vehicle License Plate Character Recognition Method Based on Artificial Neural Network", In proc. of 2nd International Asia Conference on Information in Control, Automation and Robotics, 2010, pp. 317-320.

[12] Bo Lin, Bin Fang, Dong-Hui Li, "Character Recognition Of License Plate Image Based On Multiple Classifiers" In proc. of International Conference on Wavelet Analysis and Pattern Recognition, Boading, 2009, pp. 138-143

[13] MengZe Zheng, QingYu Liu ,"Application of LVQ neural network to car license plate recognition", In Proc. of Intelligent Systems and Knowledge Engineering (ISKE), 2010, pp. 287-290.
[14] Christos Stergiou and Dimitrios Siganos [Online]. http://www.doc.ic.ac.uk/ nd/surprise_96/journal/vol4/cs11/ report.html.

[15] Jacek M. Zurada, Introduction to Artificial Neural System, West Publishing Company,10th ed. St.Paul, MN, 2004.

[16] Kishan Mehrotra, Chilukuri K. Mohan, Sanjay Ranka, Artificial Neural Networks, MIT press,1997.

[17] Kohonen, T., Self-Organization and Associative Memory, 2nd Edition, Berlin: Springer-Verlag, 1987.

[18] Martin T. Hagan, Howard B. Demuth, Mark Beale, Neural Network Design, Cengage Learning India Pvt. Ltd., $2^{\text {nd }}$ Indian reprint 2008 . 